\title{
Combination of branched-chain amino acids and angiotensin- converting enzyme inhibitor suppresses the cumulative recurrence of hepatocellular carcinoma: A randomized control trial
}

\author{
HITOSHI YOSHIJI, RYUICHI NOGUCHI, YASUHIDE IKENAKA, KOSUKE KAJI, YOSUKE AIHARA, \\ MASAHARU YAMAZAKI, JUNICHI YAMAO, MASAHISA TOYOHARA, AKIRA MITORO, MASAYOSHI SAWAI, \\ MOTOYUKI YOSHIDA, CHIE MORIOKA, MASAO FUJIMOTO, MASAHITO UEMURA and HIROSHI FUKUI
}

Third Department of Internal Medicine, Nara Medical University, Kashihara, Nara 634-8522, Japan

Received June 14, 2011; Accepted July 26, 2011

DOI: 10.3892/or.2011.1433

\begin{abstract}
Insulin resistance (IR) is reportedly involved in the progression of hepatocellular carcinoma (HCC). Since neovascularization plays an important role in hepatocarcinogenesis and IR, an angiostatic therapy may be considered as one of the promising approaches for chemoprevention against HCC. The aim of the current study was to examine the combination effect of a clinically used branched-chain amino acid (BCAA) and an angiotensin-converting enzyme inhibitor (ACE-I), both reportedly possess anti-angiogenic and IR-improving activities, on the cumulative recurrence after curative therapy. BCAA granules (Livact; $12 \mathrm{~g} /$ day) and/or ACE-I (perindopril; $4 \mathrm{mg} /$ day) were administered after the curative therapy for HCC, and several indices were analyzed. A 48-month followup revealed that the combination treatment with BCAA and ACE-I markedly inhibited the cumulative recurrence of HCC under IR conditions, whereas neither single treatment exerted a significant inhibition. The soluble form of the vascular endothelial growth factor (VEGF; a central angiogenic factor) receptor-2 (sVEGFR2) was significantly decreased only three months after the treatment without recurrence. We also observed that IR, determined by the homeostasis model
\end{abstract}

Correspondence to: Dr Hitoshi Yoshiji, Third Department of Internal Medicine, Nara Medical University, Shijo-cho 840, Kashihara, Nara 634-8522, Japan

E-mail: yoshijih@naramed-u.ac.jp

Abbreviations: EC, endothelial cells; ACE, angiotensin-converting enzyme; ACE-I, angiotensin-converting enzyme inhibitor; AFP, $\alpha$-fetoprotein; AT-II, angiotensin-II; BCAA, branched-chain amino acid; HCC, hepatocellular carcinoma; IFN, interferon; IR, insulin resistance; PIVKA-II, des- $\gamma$-carboxyprothrombin; RFA, percutaneous radiofrequency ablation; sVEGFR2, soluble form of vascular endothelial growth factor receptor-2; TACE, transarterial chemoembolization; VEGF, vascular endothelial growth factor

Key words: angiogenesis, angiotensin-converting enzyme inhibitor, branched-chain amino acid, insulin resistance, hepatocellular carcinoma assessment (HOMA-IR), was significantly improved by this regimen, indicating that an inhibitory effect was achieved, at least partly, by coordinated effects of anti-angiogenesis and IR improvement. In conclusion, since both BCAA and ACE-I are widely used in clinical practice with safety, this combination therapy may represent a potential new strategy for chemoprevention against IR-based HCC recurrence in the future. Moreover, sVEGFR2 may become a useful clinical predictive marker of this combination treatment.

\section{Introduction}

Hepatocellular carcinoma (HCC) is a malignancy of worldwide significance, and its prevalence is still increasing in Japan, Western Europe, and the United States (1-3). One of the reasons for the poor prognosis of HCC is its high rate of recurrence. Increasing evidence indicates that the high rate of recurrence, even after the curative therapy, is due to intrahepatic metastasis or multi-centric development of each respective neoplasm clone. Since the high-risk group of either primary or secondary HCC development seems to be clearer than in the other types of tumors, it is likely that chemopreventive agents will be beneficial in improving the prognosis of HCC.

Recent studies have revealed a close relationship between insulin resistance (IR, i.e., co-existence of high blood glucose and insulin levels) and the progression of liver diseases, including HCC (4,5). Cross-sectional human studies showed that IR is a consistent finding in patients with type 2 diabetes mellitus (DM), and a close interaction between DM and HCC has been documented (6). DM is associated with a 2-3-fold increase in the risk of $\mathrm{HCC}$ regardless of the etiology of chronic liver diseases $(7,8)$. Several reports have shown that IR increased the risk for the development and recurrence of HCC (7-10).

One of the characteristic features of HCC in the clinical practice is hypervascularity. Several studies have shown that neovascularization and angiogenic factors, such as the vascular endothelial growth factor (VEGF), are significantly up-regulated in the human HCC samples $(11,12)$. We previously reported that angiogenesis plays a pivotal role in the 
Table I. Demographic characteristics of the enrolled patients.

\begin{tabular}{lcccc}
\hline Characteristics & Control $(\mathrm{G} 1)$ & Combination $(\mathrm{G} 2)$ & ACE-I $(\mathrm{G} 3)$ & BCAA (G4) \\
\hline No. of patient & 26 & 28 & 19 & 16 \\
Age (years) & $62.5 \pm 11.5^{\mathrm{a}}$ & $64.8 \pm 10.6(0.338)^{\mathrm{b}}$ & $59.4 \pm 12.3(0.390)$ & $63.7 \pm 10.8(0.409)$ \\
Gender (M/F) & $16 / 10$ & $18 / 10(0.888)$ & $12 / 7(0.911)$ & $10 / 6(0.950)$ \\
Etiology (HCV/HBV/other) & $18 / 6 / 2$ & $19 / 8 / 1(0.5)$ & $14 / 4 / 1(0.208)$ & $10 / 5 / 1(0.353)$ \\
Alcohol $(<40 \mathrm{~g} />40 \mathrm{~g} / \mathrm{day})$ & $20 / 6$ & $22 / 6(0.884)$ & $12 / 7(0.314)$ & $12 / 4(0.887)$ \\
Tumor stage (I/II/III) & $18 / 7 / 1$ & $22 / 5 / 1(0.454)$ & $12 / 6 / 1(0.665)$ & $10 / 5 / 1(0.637)$ \\
Tumor mean size (mm) & $20.7 \pm 8.5$ & $19.6 \pm 9.6(0.267)$ & $20.1 \pm 8.0(0.401)$ & $18.1 \pm 9.0(0.419)$ \\
AFP (ng/ml) & $75.3 \pm 253.3$ & $82.3 \pm 282.5(0.289)$ & $92.4 \pm 303.1(0.218$ & $79.4 \pm 273.6(0.386)$ \\
ALT (IU/l) & $78.8 \pm 31.5$ & $72.3 \pm 40.6(0.099)$ & $74.8 \pm 36.6(0.258)$ & $69.4 \pm 29.6(0.411)$ \\
HOMA-IR & $3.79 \pm 2.92$ & $4.15 \pm 2.76(0.386)$ & $3.28 \pm 3.42(0.247)$ & $3.64 \pm 2.96(0.492)$ \\
FBS (mg/dl) & $113.4 \pm 28.8$ & $106.5 \pm 31.4(0.329)$ & $94.6 \pm 28.8(0.509)$ & $103.8 \pm 35.8(0.191)$ \\
Child-Pugh (A/B) & $21 / 5$ & $22 / 6(0.841)$ & $16 / 3(0.765)$ & $12 / 4(0.658)$ \\
\end{tabular}

${ }^{\mathrm{a}}$ The data represent mean $\pm \mathrm{SD}$. ${ }^{\mathrm{b}} \mathrm{P}$-value as compared with the control group.

murine HCC development, and that suppression of VEGFsignaling pathway markedly attenuated the HCC growth and hepatocarcinogenesis $(13,14)$. It has been reported that the IR status itself directly accelerated hepatocarcinogenesis via stimulation of hepatic neovascularization (4), indicating that any agent with both anti-angiogenic and IR-improving effects would be a useful tool for HCC chemoprevention under the condition of IR.

In the clinical practice, several anti-angiogenic agents have already been employed including sorafenib (15). Sorafenib is an oral multi-kinase inhibitor that acts against several tyrosine kinases (VEGFR1, PDGFR, c-kit receptors) and serine/ threonine kinases (b-Raf, p38) (16). It has been reported that sorafenib prolonged the overall survival and delayed the median time to progression in patients with advanced HCC, and that this drug would become the standard of cure for advanced HCC (17). However, there are apparently several serious concerns in employing this agent for chemoprevention against HCC. As long-term administration is required and the drug metabolism is usually hypoactive in the patients with cirrhosis, an agent with proven safety would be preferable for chemoprevention against HCC. Almost all patients show adverse effects on using sorafenib, and several symptoms are very severe $(18,19)$. Moreover, considering the long-term administration, cost-performance is a problem since this drug is very expensive (20). An alternative approach may be to find a clinically available compound that also exhibits anti-angiogenic activity for which the safety of long-term administration has been proven.

The branched-chain amino acids (BCAA) comprise three essential amino acids; namely, leucine, iso-leucine, and valine. Recent studies have shown that long-term supplementation with BCAA granules (a mixture of these three amino acids) to patients with liver cirrhosis improved the nutritional status, such as hypoalbuminemia, and event-free survival $(21,22)$. BCAA suppressed the incidence of hepatocarcinogenesis in patients with HCV-related cirrhosis and obesity that is often associated with IR (23). We have recently reported that BCAA exerted a chemopreventive effect against HCC along with suppression of VEGF expression and hepatic neovascularization in obese diabetic rats having IR (24). We also previously reported that the clinically used an angiotensin-converting enzyme inhibitor (ACE-I) exhibited a strong anti-angiogenic activity, and that ACE-I showed suppressive effect against hepatocarcinogenesis via angiogenesis suppression $(25,26)$. In addition, both BCAA and ACE-I reportedly improve IR in the clinical practice $(27,28)$. It has been reported that combination treatments of anti-angiogenic agents exert a more potent inhibitory effect on the tumor development than single-agent treatment (29). We previously reported that the combination treatment of BCAA and ACE-I at clinically comparable low doses showed a marked suppressive effect against development of HCC in rats under the condition of IR via angiogenesis suppression as compared with either single agent $(24,30)$.

In the current study, we examined whether the combination of BCAA and ACE-I suppressed the disease recurrence in patients with HCC under the condition of IR after they had received curative therapy.

\section{Patients and methods}

Patients. This study was conducted on 110 patients with HCC who were admitted to our hospital between May 2004 and July 2006. HCC was diagnosed with combination of several imaging modalities; namely, ultrasonography (US), computer tomography (CT), magnetic resonance imaging (MRI), and hepatic angiography. Since we did not encounter any unusual lesions in the current study that require needle biopsy for histological confirmation, we did not have any histological proof of the diagnosis of our patients. All patients received curative therapy with percutaneous radiofrequency ablation (RFA) for prior HCC, and were confirmed to be free of any residual HCC by several imaging modalities. The IR index was calculated on the basis of fasting values for glycemia and insulinemia, according to the homeostasis model assessment (HOMA-IR) method as previously described (31). We also 


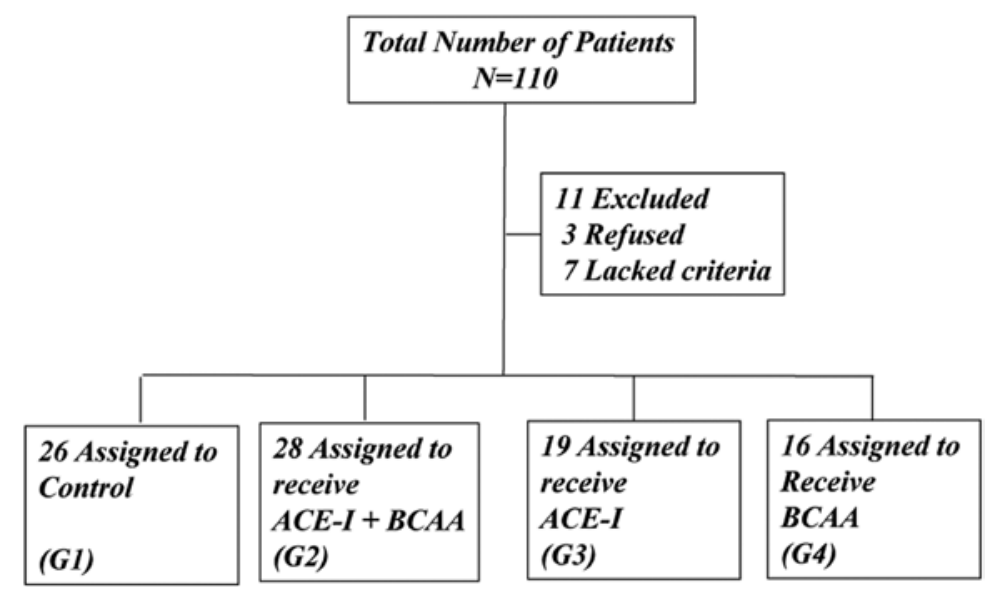

Figure 1. The study design profile for evaluation of the effects of ACE-I and BCAA on the cumulative recurrence after curative therapy under the condition of IR. Among the enrolled 110 patients, 89 were randomly divided into the control group (Group 1, G1: $\mathrm{n}=26$ ), combination-treated group (Group 2, G2: $\mathrm{n}=25$ ), ACE-I-treated group (Group 3, G3: n=19), and BCAA-treated group (Group 4, G4: n=16) continuously for 48 months. We could follow-up all enrolled patients in each group for analysis.

performed the 75-g-oral glucose tolerance test (OGTT), and a certified doctor of Japan Diabetes Society confirmed the status of IR of the enrolled patients. The clinical profiles, laboratory data, and characteristics of the patients are shown in Table I. We recommended all patients to stop alcohol intake completely. Until the end of the follow-up for recurrence, no patients received any additional therapy for HCC, such as interferon (IFN). All patients gave written informed consent before participating in this study. This protocol was approved by the Institutional Review Board (IRB) of Nara Medical University, and the study was conducted in conformity with the ethical and humane principles. We carefully followed the CONSORT Statement for randomized studies to perform the current analysis.

Study design. In the current study, we employed the therapeutic modalities according to the algorithm of HCC treatment by the Liver Cancer Study Group of Japan (LCSGJ). The LCSGJ scoring system is a reliable system that is comparable to those of the Cancer of the Liver Italian Program (CLIP), the Barcelona Clinic Liver Center (BCLC), and the evidencebased clinical practice guidelines for HCC in Japan supported by the Japanese Ministry of Health, Labor, and Welfare $(2,32-34)$. In this algorithm, the therapeutic strategy is chosen based on the degree of liver damage as determined by LCSGJ and the characteristics of the tumor itself. The indications of RFA of LCSGJ are three or fewer tumors measuring $3 \mathrm{~cm}$ or less, or a solitary tumor with a major axis of $5 \mathrm{~cm}$ or less. In our university, an inter-department conference is held every week. We discuss the selection of therapeutic modalities with surgeons and radiologists in each case of HCC. When RFA is selected in this conference, the patient is admitted into our department. After RFA, follow-up was conducted using enhanced CT and US once a month for the first three months. If any viable $\mathrm{HCC}$ was detected during this period, the patient was excluded from the current study.

As shown in the demographics of the current study (Fig. 1), we enrolled 110 patients. Among them, we could follow up 89 patients. The patients were randomly divided by sealed envelope into either the control group (Group 1, G1: $n=26$ ), combination-treated group (Group 2, G2: $\mathrm{n}=28$ ), either single treatment with ACE-I (perindopril; $4 \mathrm{mg} /$ day) or BCAA granules (Livact; $12 \mathrm{~g}$ /day) (G3: n=19 and G4: n=16, respectively) continuously for 48 months. The respective dose of both compounds is a standard dose in the clinical practice.

Detection of HCC recurrence. Follow-up was conducted for all patients every 4 weeks at our hospital. To find the recurrent HCC nodules, imaging studies such as US and CT were performed every 3 months. The serum tumor markers; namely, $\alpha$-fetoprotein (AFP), and des- $\gamma$-carboxyprothrombin (PIVKA-II), were measured every 2 months with routine laboratory methods. Prior to and at 12 months after starting drug administration, the alterations of angiogenic factors were checked using a TranSignal Angiogenesis Antibody Array (Panomics Inc., Redwood City, CA, USA) in the serum according to the manufacturer's manual after equalization of the protein content. We also examined the alteration of VEGF (35), sVEGFR1 and sVEGFR2 in the serum using an enzyme-linked immunosorbent assay (ELISA) kit (R\&D Systems), according to the manufacturer's instructions as previously described (14). When any recurrence was detected, the patient was excluded from the protocol. Immediately, such patients were treated as having secondary HCC according to the algorithm of LCSGJ. We conducted follow-up every month during the observation period, and all recurrent cases met the aforementioned RFA criteria (namely, three or fewer tumors, $<3 \mathrm{~cm}$ or a solitary tumor, $<5 \mathrm{~cm}$ ). Accordingly, all patients with the first recurrence were treated with RFA. Nevertheless, several patients with a secondary recurrence received transarterial chemoembolization (TACE) and/or arterial infusion chemotherapy afterward. Liver transplantation is uncommon in Japan for patients with recurrent HCC.

Statistical analysis. The variables of the characteristics of enrolled patients were analyzed with the Mann-Whitney U test and the Fisher exact probability test. The cumulative recurrence of HCC was plotted using the Kaplan-Meier method, 


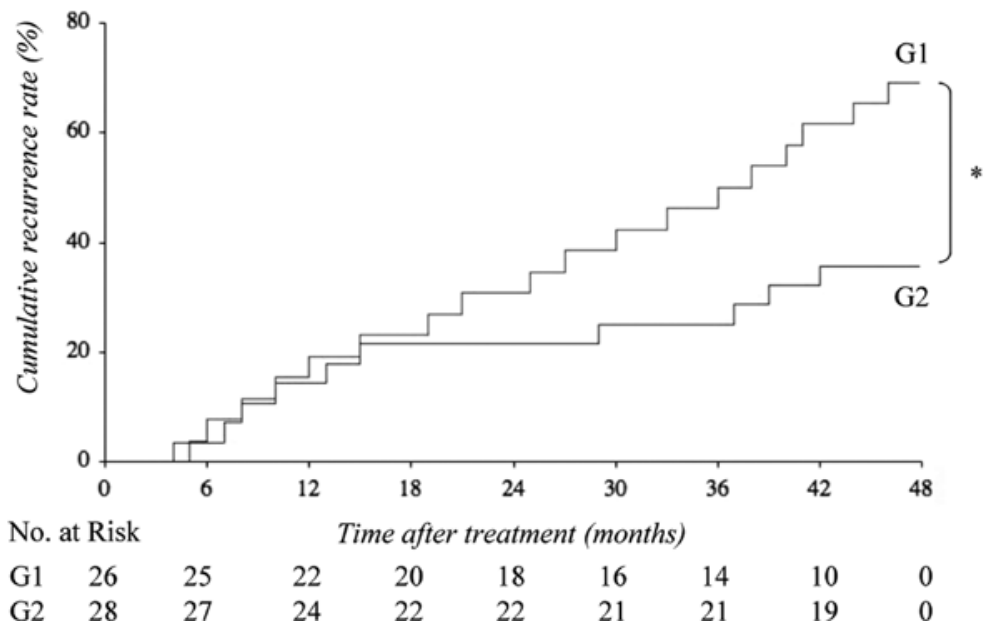

Figure 2. The cumulative recurrence of secondary HCC after curative therapy by the combination treatment of ACE-I and BCAA under the condition of IR for 48 months. The combination treatment of ACE-I and BCAA $(G 2, n=28)$ significantly suppressed the cumulative recurrence of HCC as compared with the control group ( $\mathrm{G} 1, \mathrm{n}=26)$. "Statistically significant differences between the indicated groups $(\mathrm{P}<0.01)$.

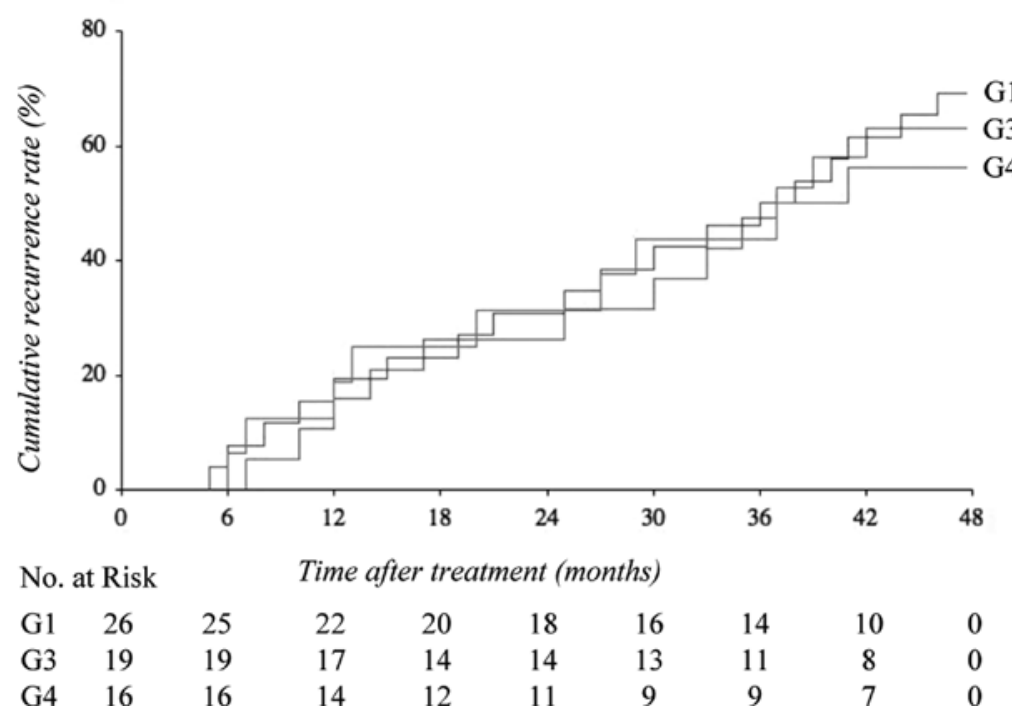

Figure 3. The cumulative recurrence of secondary HCC after curative therapy by single treatment with either ACE-I or BCAA for 48 months. Single treatment with either ACE-I (G3, n=19) or BCAA (G4, n=16) did not exert any inhibitory effect on the cumulative recurrence of HCC as compared with the control group $(\mathrm{G} 1, \mathrm{n}=26)$.

and the differences in the recurrence curves were tested using the log-rank test.

\section{Results}

Patient characteristics. The characteristics of the enrolled patients are shown in Table I. There were no significant differences among the patients of all groups in age, gender, etiology of the disease, or background liver function (Child-Pugh score). Also, the tumor baseline, such as stage, number of tumors, AFP, PIVKA-II levels, did not differ among the groups. Both ACE-I and BCAA are widely used without serious adverse effects, and the safety of their long-term administration has been proven in the clinical practice. In the current study, there were no severe toxic effects in any group, and no abnormal laboratory data were found that could likely be related to treatment with either ACE-I or BCAA. Accordingly, after randomization, we could follow up all the enrolled patients in each group until detection of recurrence.

HCC recurrence. The results of combination treatment of BCAA and ACE-I are shown in Fig. 2. The combination treatment of BCAA and ACE-I (G2) significantly suppressed the cumulative recurrence of $\mathrm{HCC}$ as compared with the control group (G1) for 48 months after the treatment $(\mathrm{P}<0.01)$. On the other hand, single treatment with either ACE-I (G3) or BCAA (G4) did not exert any inhibitory effect on the cumulative recurrence of HCC as compared with the control group (G1) (Fig. 3). We compared several markers among the enrolled patients following the 12-month treatment (Table II). The mean blood pressure decreased in the combination- and ACE-I-treated groups, but there was no significant difference 
Table II. Changes of several markers in the enrolled patients following 12-month treatment.

\begin{tabular}{|c|c|c|c|c|c|c|c|c|}
\hline & \multicolumn{2}{|c|}{ Control (G1) } & \multicolumn{2}{|c|}{ Combination (G2) } & \multicolumn{2}{|c|}{ ACE-I (G3) } & \multicolumn{2}{|c|}{$\mathrm{BCAA}(\mathrm{G} 4)$} \\
\hline & Before & After & Before & After & Before & After & Before & After \\
\hline Mean BP (mmHg) & $138 \pm 21^{\mathrm{a}}$ & $132 \pm 18$ & $140 \pm 36$ & $120 \pm 25^{b}$ & $138 \pm 29$ & $120 \pm 17^{b}$ & $143 \pm 16$ & $136 \pm 18$ \\
\hline $\mathrm{AFP}(\mathrm{ng} / \mathrm{ml})$ & $75.3 \pm 253.3$ & $91.2 \pm 403.6^{\mathrm{b}}$ & $82.3 \pm 268.2$ & $62.4 \pm 185.6$ & $90.8 \pm 362.2$ & $84.8 \pm 326.3$ & $78.8 \pm 194.8$ & $70.2 \pm 176.6$ \\
\hline PIVKA-II (mAU/ml) & $38.6 \pm 70.3$ & $51.6 \pm 62.2$ & $63.1 \pm 58.2$ & $51.2 \pm 48.9$ & $57.3 \pm 50.4$ & $48.7 \pm 50.2$ & $61.4 \pm 52.2$ & $53.8 \pm 44.2$ \\
\hline VEGF (ng/ml) & $104.2 \pm 52.3$ & $148.6 \pm 36.5^{\mathrm{b}}$ & $110.6 \pm 49.7$ & $83.2 \pm 33.2^{\mathrm{b}}$ & $98.7 \pm 36.8$ & $80.3 \pm 39.2$ & $104.6 \pm 40.1$ & $85.4 \pm 36.3$ \\
\hline sVEGFR2 (pg/ml) & $7204 \pm 1015$ & $8972 \pm 650^{b}$ & $7421 \pm 1719$ & $4824 \pm 1024^{\mathrm{c}}$ & $6894 \pm 1521$ & $5620 \pm 1051$ & $7016 \pm 11810$ & $6101 \pm 1728$ \\
\hline Alb (g/dl) & $3.6 \pm 1.2$ & $3.5 \pm 1.3$ & $3.3 \pm 1.2$ & $3.7 \pm 1.4$ & $3.4 \pm 1.4$ & $3.4 \pm 1.3$ & $3.4 \pm 1.2$ & $3.8 \pm 1.5$ \\
\hline ALT (IU/l) & $74.3 \pm 30.6$ & $70.8 \pm 28.8$ & $69.6 \pm 31.3$ & $60.7 \pm 36.6$ & $76.6 \pm 33.4$ & $62.8 \pm 34.4$ & $70.8 \pm 29.7$ & $64.3 \pm 28.1$ \\
\hline FBS (mg/dl) & $113.4 \pm 28.8$ & $107.8 \pm 31.2$ & $106.5 \pm 31.4$ & $98.6 \pm 27.5$ & $99.8 \pm 27.6$ & $92.7 \pm 28.9$ & $102.7 \pm 30.6$ & $95.4 \pm 31.1$ \\
\hline HOMA-R & $3.79 \pm 2.92$ & $3.61 \pm 2.88$ & $4.15 \pm 2.76$ & $2.82 \pm 1.94^{\mathrm{b}}$ & $3.88 \pm 2.79$ & $3.14 \pm 2.27$ & $3.55 \pm 3.01$ & $2.75 \pm 2.08$ \\
\hline
\end{tabular}

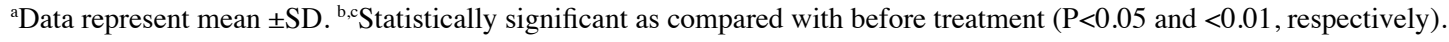

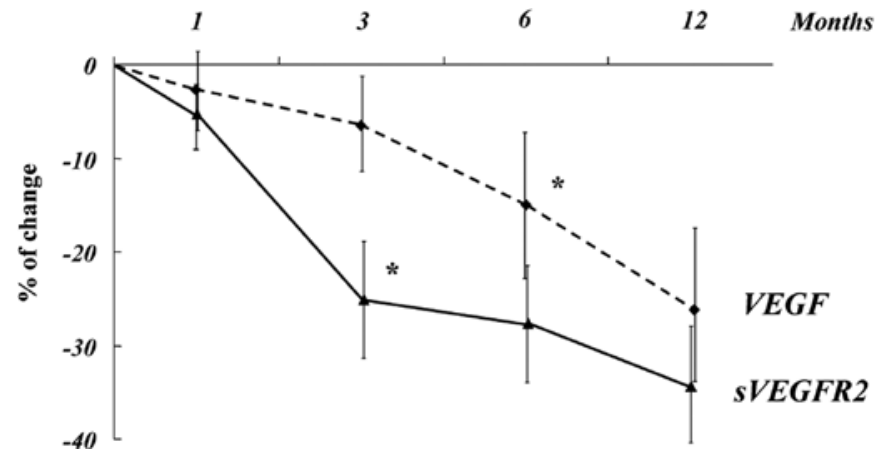

Figure 4. The chronological alterations of VEGF and sVEGFR2 in the treated group until 12 months after treatment in the patients without $\mathrm{HCC}$ recurrence. The VEGF expression gradually decreased, and the expression level at 6 months after the treatment was significantly lower than the pretreatment basal level. The decrease of sVEGFR2 expression was earlier than that of VEGF. The sVEGFR2 expression already significantly decreased at three months after the ACE-I and BCAA combination treatment. The data represent means $\pm \mathrm{SD}(\mathrm{n}=28)$. "Statistically significant differences as compared with the pre-treatment basal level $(\mathrm{P}<0.01)$.

between the groups. Furthermore, there were no significant differences in the ALT level between the combination-treated and other groups, indicating that the suppressive effect of this combination treatment on the cumulative recurrence was not due to anti-hypertensive or cytoprotective activities. We next examined whether or not the alteration of AFP and PIVKA-II levels correlated with the inhibitory effect on the cumulative recurrence of $\mathrm{HCC}$ in the combination-treated group. The AFP level was significantly decreased during the 12-month period of administration in the combination-treated, whereas the PIVKA-II level did not show any marked reduction. Although the primary end-point of the current study was the cumulative recurrence rates, we also examined whether the survival curves of the patients were altered or not during the follow-up period. We found no statistical differences between the groups (data not shown).

Alteration of angiogenic factors and IR. To elucidate which angiogenic factors were altered by treatment with ACE-I and
BCAA, we employed the angiogenic antibody array that can detect 19 different angiogenic factors in the serum of the enrolled patients following 12-month treatment as previously described (36). We performed this array analysis using fivepaired set of samples ( $\mathrm{n}=10$ in total). In the control group, the spot of VEGF was significantly increased, whereas it was remarkably attenuated by the combined treatment of ACE-I and BCAA. A recent research has shown that the HGF level may become a biomarker of sorafenib clinical efficiency. However, in our ACE-I and BCAA combination treatment, no significant alteration of HGF could be detected (data not shown).

Since the VEGF expression was predominantly changed by ACE-I and BCAA combination treatment, we measured the expression levels of the VEGF-related molecules by ELISA in all patients. The serum VEGF level in the control group increased after 12 months, whereas the combination treatment of ACE-I and BCAA markedly attenuated the VEGF level as compared with the pre-treatment level (Table II). We also elucidated the expression levels of sVEGFR2. As shown in Table II, sVEGFR2 was markedly decreased by the combination treatment of ACE-I and BCAA whereas the serum level of sVEGFR2 in the control group increased. On the other hand, there was no significant difference in sVEGFR1 between the pre-treatment and post-treatment levels in either group (data not shown). We next examined the chronological alterations of VEGF and sVEGFR2 in the treated group until 12 months after the treatment in patients without HCC recurrence. The VEGF expression level gradually decreased, but at 6-month treatment it was statistically significant as compared with the pre-treatment basal level. The decrease of sVEGFR2 expression was earlier than that of VEGF. The sVEGFR2 expression already significantly decreased at three months after the ACE-I and BCAA combination treatment (Fig. 4).

We also examined the alteration of IR status by the HOMA-IR score. As shown in the Table II, the combination treatment with BCAA and ACE-I for 12 months significantly decreased the median HOMA-IR score. Single treatment of BCAA or ACE-I also tended to decrease the HOMA-IR level, although neither of them showed significance. 


\section{Discussion}

In the current study, we found that treatment with the clinically used BCAA and ACE-I markedly inhibited the cumulative HCC recurrence after curative therapy under the condition of IR along with suppression of several indices. Monotherapy with ACE-I or BCAA did not exert any inhibitory effect, either. This is consistent with a previous report showing that BCAA monotherapy failed to suppress the first and second recurrence of HCC after the curative therapy in the clinical practice (37). Several recent studies have suggested that treatment using a single anti-angiogenic agent may not be sufficient to completely inhibit tumor angiogenesis $(38,39)$. Recently, it has been reported that although the anti-angiogenic activity of single agents was negligible, the combination treatment with different angiostatic agents showed remarkably synergistic inhibitory effects on pathological neovascularization (40). Since the actions of ACE-I and BCAA are mediated via different anti-angiogenic pathways (41), it is possible that the coordination of these different biological activities produced the combined suppressive effect of ACE-I and BCAA; an effect that is not secured when either single agent is used.

We observed that the inhibitory effects of BCAA and ACE-I correlated with the decrease of the angiogenic factor, VEGF. We previously reported that both BCAA and ACE-I could suppress the VEGF expression during hepatocarcinogenesis in rats $(24,42)$. Nevertheless, there seems to be a different mechanism for VEGF suppression between the two agents. Angiotensin-II induces VEGF gene expression and promoter activation via hypoxia-inducible factor-1 (HIF-1), that is one of the main VEGF gene activators (43). On the other hand, the iso-leucine-mediated VEGF suppression is independent of HIF-1, but it is achieved via inhibition of the mammalian target of rapamycin (mTOR) (44). It is likely that the coordination of these different biological activities of anti-angiogenic agents produced the combination effect of suppressing hepatocarcinogenesis.

We also observed that sVEGFR2 significantly decreased, and the reduction of sVEGFR2 appeared earlier than that of VEGF just after 3 months treatment in the patients without recurrence. It is very important to find surrogate markers that can predict the clinical outcome of anti-angiogenic therapies, since several currently available anti-angiogenic agents exert severe diverse effects. We previously reported that the VEGF and VEGFR2 interaction plays a pivotal role in HCC growth and hepatocarcinogenesis $(14,45)$. Recent clinical studies have evaluated the plasma levels of sVEGFR2 as a potential surrogate marker of disease progression in various malignancies based on the hypothesis that the circulating sVEGFR2 level may provide insight into the VEGFR2 activation (46). We observed that the expressions of VEGF and sVEGFR2 chronologically decreased by the combined treatment of ACE-I and BCAA in the patients without $\mathrm{HCC}$ recurrence. The decrease of sVEGFR2 expression was earlier than that of VEGF. The sVEGFR 2 expression already significantly decreased even at three months after the ACE-I and BCAA combination treatment. In renal cell carcinoma, the sVEGFR2 levels significantly decreased by treatment with sunitinib, which is also a multikinase inhibitor, including VEGFR, during the treatment cycle
1 (47). Moreover, the levels tended to return to near baseline after 2 weeks off treatment, indicating that sVEGFR2 was possibly a sensitive marker of anti-angiogenic therapy. These results indicated that sVEGFR2 may be utilized as a useful clinical predictive marker of this combination treatment of ACE-I and BCAA. In HCC, it has been found that sVEGFR1 correlated with poor prognosis, whereas it was not an independent prognostic factor for HCC (48). In the current study, we did not find any significant correlation between the sVEGFR1 alteration and HCC recurrence, either.

BCAA and ACE-I are known to exert multiple pharmacological activities. We observed that IR was significantly improved by the combination treatment. This effect is also likely to play a critical role in the clinical beneficial effect since insulin is now recognized as a multi-functional protein involved in the tumor cell proliferation and anti-apoptotic activity (4). It has been reported that BCAA improved glucose tolerance by modulating the insulin-independent glucose uptake (49). ACE-I can also improve the glucose tolerance by several mechanisms, such as inducing glucose transporter 4 (GLUT4) or inhibition of suppressor of cytokine signaling 3 (SOCS3) (28). We did not find a marked alteration of FBS level in the current study. However, in some pilot cases, we observed that the combined treatment improved the pattern of oral glucose tolerance test (data not shown). These coordinating different activities should contribute to the inhibitory effect of this combination regimen. Further studies are required to elucidate the exact role of each activity in the future.

Although the primary end-point of the current study was the cumulative recurrence rates, we also examined whether the survival curves of the patients were altered or not during the follow-up period. No statistical differences could be found among the groups (data not shown). Since the follow-up period in the current study was probably not long enough, no statistical differences could be detected among the groups. Further long-term and large-scale studies are also required to verify whether or not the suppressive effects of ACE-I and BCAA on the cumulative recurrence will improve the prognosis in the future.

In conclusion, we have shown that the combination treatment with clinically used safe agents; namely, BCAA and ACE-I, markedly inhibited the cumulative HCC recurrence after curative therapy under the condition of IR along with suppression of several indices such as VEGF, sVEGFR2 and HOMA-IR. Although a large-scale long-term follow-up clinical control study is required, this combined treatment may represent a potential new strategy for secondary chemoprevention of HCC, and sVEGFR-2 could become a useful clinical predictive marker of this combination treatment.

\section{References}

1. Aravalli RN, Steer CJ and Cressman EN: Molecular mechanisms of hepatocellular carcinoma. Hepatology 48: 2047-2063, 2008.

2. Arii S, Sata M, Sakamoto M, et al: Management of hepatocellular carcinoma: Report of Consensus Meeting in the 45th Annual Meeting of the Japan Society of Hepatology (2009). Hepatol Res 40: 667-685, 2010.

3. Minguez B, Tovar V, Chiang D, Villanueva A and Llovet JM: Pathogenesis of hepatocellular carcinoma and molecular therapies. Curr Opin Gastroenterol 25: 186-194, 2009. 
4. Kaji K, Yoshiji H, Kitade M, et al: Impact of insulin resistance on the progression of chronic liver diseases. Int J Mol Med 22: 801-808, 2008

5. Llovet JM and Bruix J: Novel advancements in the management of hepatocellular carcinoma in 2008. J Hepatol 48 (Suppl 1): S20-S37, 2008

6. Kahn CR: Insulin resistance, insulin insensitivity, and insulin unresponsiveness: a necessary distinction. Metabolism 27: 1893-1902, 1978.

7. Davila JA, Morgan RO, Shaib Y, McGlynn KA and El-Serag HB Diabetes increases the risk of hepatocellular carcinoma in the United States: a population based case control study. Gut 54: 533-539, 2005.

8. El-Serag HB, Tran T and Everhart JE: Diabetes increases the risk of chronic liver disease and hepatocellular carcinoma. Gastroenterology 126: 460-468, 2004

9. El-Serag HB: Epidemiology of hepatocellular carcinoma in USA. Hepatol Res 37 (Suppl 2): S88-S94, 2007.

10. Imai K, Takai K, Nishigaki Y, et al: Insulin resistance raises the risk for recurrence of stage I hepatocellular carcinoma after curative radiofrequency ablation in hepatitis $\mathrm{C}$ viruspositive patients: a prospective, case series study. Hepatol Res 40: 376-382, 2010

11. Mise M, Arii S, Higashituji $\mathrm{H}$, et al: Clinical significance of vascular endothelial growth factor and basic fibroblast growth factor gene expression in liver tumor. Hepatology 23: 455-464, 1996.

12. Yamaguchi R, Yano H, Iemura A, Ogasawara S, Haramaki M and Kojiro M: Expression of vascular endothelial growth factor in human hepatocellular carcinoma. Hepatology 28: 68-77, 1998

13. Yoshiji H, Kuriyama S, Hicklin DJ, et al: KDR/Flk-1 is major regulator of vascular endothelial growth factor- induced tumor development and angiogenesis in murine hepatocellular carcinoma cells. Hepatology 30: 1179-1186, 1999.

14. Yoshiji H, Kuriyama S, Yoshii J, et al: Halting the interaction between vascular endothelial growth factor and its receptors attenuates liver carcinogenesis in mice. Hepatology 39: 1517-1524, 2004

15. Kerbel RS: Tumor angiogenesis. N Engl J Med 358: 2039-2049, 2008.

16. Wilhelm SM, Carter C, Tang L, et al: BAY 43-9006 exhibits broad spectrum oral antitumor activity and targets the RAF/ MEK/ERK pathway and receptor tyrosine kinases involved in tumor progression and angiogenesis. Cancer Res 64: 7099-7109, 2004.

17. Llovet JM, Ricci S, Mazzaferro V, et al: Sorafenib in advanced hepatocellular carcinoma. N Engl J Med 359: 378-390, 2008.

18. Eskens FA and Verweij J: The clinical toxicity profile of vascular endothelial growth factor (VEGF) and vascular endothelia growth factor receptor (VEGFR) targeting angiogenesis inhibitors: a review. Eur J Cancer 42: 3127-3139, 2006

19. Verheul HM and Pinedo HM: Possible molecular mechanisms involved in the toxicity of angiogenesis inhibition. Nat Rev Cancer 7: 475-485, 2007.

20. Berenson A: A cancer drug shows promise, at a price that many can't pay. NY Times (Print) A1, C2, 2006

21. Marchesini G, Bianchi G, Merli M, et al: Nutritional supplementation with branched-chain amino acids in advanced cirrhosis: a double-blind, randomized trial. Gastroenterology 124: 1792-1801, 2003

22. Muto Y, Sato S, Watanabe A, et al: Effects of oral branchedchain amino acid granules on event-free survival in patients with liver cirrhosis. Clin Gastroenterol Hepatol 3: 705-713, 2005.

23. Muto Y, Sato S, Watanabe A, et al: Overweight and obesity increase the risk for liver cancer in patients with liver cirrhosis and long-term oral supplementation with branched-chain amino acid granules inhibits liver carcinogenesis in heavier patients with liver cirrhosis. Hepatol Res 35: 204-214, 2006.

24. Yoshiji H, Noguchi R, Kitade M, et al: Branched-chain amino acids suppress insulin-resistance-based hepatocarcinogenesis in obese diabetic rats. J Gastroenterol 44: 483-491, 2009.

25. Yoshiji H, Kuriyama S, Noguchi R and Fukui H: Angiotensin-I converting enzyme inhibitors as potential anti-angiogenic agents for cancer therapy. Curr Cancer Drug Targets 4: 555-567, 2004.

26. Yoshiji H, Noguchi R, Ikenaka Y, et al: Renin-angiotensin system inhibitors as therapeutic alternatives in the treatment of chronic liver diseases. Curr Med Chem 14: 2749-2754, 2007.

27. Kawaguchi T, Yamagishi S and Sata M: Branched-chain amino acids and pigment epithelium-derived factor: novel therapeutic agents for hepatitis c virus-associated insulin resistance. Curr Med Chem 16: 4843-4857, 2009.
28. Muscogiuri G, Chavez AO, Gastaldelli A, et al: The crosstalk between insulin and renin-angiotensin-aldosterone signaling systems and its effect on glucose metabolism and diabetes prevention. Curr Vasc Pharmacol 6: 301-312, 2008

29. Kerbel RS: Clinical trials of antiangiogenic drugs: opportunities, problems, and assessment of initial results. J Clin Oncol 19: S45-S51, 2001

30. Yoshiji H, Noguchi R, Kaji K, et al: Attenuation of insulinresistance-based hepatocarcinogenesis and angiogenesis by combined treatment with branched-chain amino acids and angiotensin-converting enzyme inhibitor in obese diabetic rats. J Gastroenterol 45: 443-450, 2010.

31. Fartoux L, Poujol-Robert A, Guechot J, Wendum D, Poupon R and Serfaty L: Insulin resistance is a cause of steatosis and fibrosis progression in chronic hepatitis C. Gut 54: 1003-1008, 2005.

32. Kokudo N and Makuuchi M: Evidence-based clinical practice guidlines for hepatocellular carcinoma in Japan: the J-HCC guidlines. J Gastroenterol 44: 119-121, 2009.

33. Makuuchi M, Kokudo N, Arii S, et al: Development of evidencebased clinical guidelines for the diagnosis and treatment of hepatocellular carcinoma in Japan. Hepatol Res 38: 37-51, 2008.

34. Okita K: Management of hepatocellular carcinoma in Japan. J Gastroenterol 41: 100-106, 2006.

35. Ferrara N: VEGF as a therapeutic target in cancer. Oncology 69 (Suppl 3): 11-16, 2005.

36. Yoshiji H, Noguchi R, Toyohara M, et al: Combination of vitamin K2 and angiotensin-converting enzyme inhibitor ameliorates cumulative recurrence of hepatocellular carcinoma. J Hepatol 51: 315-321, 2009.

37. Tsuchiya K, Asahina Y, Sato T, et al: Oral supplementation with branched-chain amino acid (BCAA) improves survival and dcreases the third time recurrence after successful treatment of hepatocellular carcinoma (HCC). J Hepatol (EASL proceeding) 48 (suppl No. 2): S160, 2009.

38. Kerbel RS and Kamen BA: The anti-angiogenic basis of metronomic chemotherapy. Nat Rev Cancer 4: 423-436, 2004.

39. Davis DW, Inoue K, Dinney CP, Hicklin DJ, Abbruzzese JL and McConkey DJ: Regional effects of an antivascular endothelial growth factor receptor monoclonal antibody on receptor phosphorylation and apoptosis in human 253J B-V bladder cancer xenografts. Cancer Res 64: 4601-4610, 2004.

40. Dorrell MI, Aguilar E, Scheppke L, Barnett FH and Friedlander M: Combination angiostatic therapy completely inhibits ocular and tumor angiogenesis. Proc Natl Acad Sci USA 104: 967-972, 2007.

41. Yoshiji H, Kuriyama S, Kawata M, et al: The angiotensin-Iconverting enzyme inhibitor perindopril suppresses tumor growth and angiogenesis: possible role of the vascular endothelial growth factor. Clin Cancer Res 7: 1073-1078, 2001.

42. Yoshiji H, Yoshii J, Ikenaka Y, et al: Inhibition of renin-angiotensin system attenuates liver enzyme-altered preneoplastic lesions and fibrosis development in rats. J Hepatol 37: 22-30, 2002.

43. Sanchez-Lopez E, Lopez AF, Esteban V, et al: Angiotensin II regulates vascular endothelial growth factor via hypoxia-inducible factor-lalpha induction and redox mechanisms in the kidney. Antioxid Redox Signal 7: 1275-1284, 2005.

44. Murata K and Moriyama M: Isoleucine, an essential amino acid, prevents liver metastases of colon cancer by antiangiogenesis. Cancer Res 67: 3263-3268, 2007.

45. Yoshiji H, Kuriyama S, Yoshii J, et al: Synergistic effect of basic fibroblast growth factor and vascular endothelial growth factor in murine hepatocellular carcinoma. Hepatology 35: 834-842, 2002.

46. Wierzbowska A, Robak T, Wrzesien-Kus A, Krawczynska A, Lech-Maranda E and Urbanska-Rys H: Circulating VEGF and its soluble receptors sVEGFR-1 and sVEGFR-2 in patients with acute leukemia. Eur Cytokine Netw 14: 149-153, 2003.

47. Deprimo SE, Bello CL, Smeraglia J, et al: Circulating protein biomarkers of pharmacodynamic activity of sunitinib in patients with metastatic renal cell carcinoma: modulation of VEGF and VEGF-related proteins. J Transl Med 5: 32, 2007.

48. Nagaoka S, Yoshida T, Akiyoshi J, et al: The ratio of serum placenta growth factor to soluble vascular endothelial growth factor receptor-1 predicts the prognosis of hepatocellular carcinoma. Oncol Rep 23: 1647-1654, 2010.

49. Nishitani S, Takehana K, Fujitani S and Sonaka I: Branchedchain amino acids improve glucose metabolism in rats with liver cirrhosis. Am J Physiol Gastrointest Liver Physiol 288: G1292-G1300, 2005. 